Peer-reviewed research

\title{
Investor Sentiment and Volatility Prediction of Currencies and Commodities During the COVID-19 Pandemic
}

\author{
Thi Hong Van Hoang ${ }^{1}$, Qasim Raza Syed ${ }^{2}$ \\ ${ }^{1}$ Montpellier Business School, 2300 avenue des Moulins, Montpellier, France, ${ }^{2}$ National Tariff Commission, Islamabad, Pakistan \\ Keywords: gfc, commodities, currencies, volatility prediction, investor sentiment, covid-19 \\ 10.46557/001c.18642
}

\section{Asian Economics Letters}

In this note, we examine whether the volatility predictive power of investor sentiment for currencies and commodities is sensitive to the COVID-19 pandemic. The Credit Suisse Fear Barometer (CSFB) and the VIX are used to measure investor sentiment. The volatility of seven major currencies, gold, and oil is investigated. Using daily data from 2005 to 2020, we show that VIX is a better predictor than CSFB. However, they have no predictive power during the COVID-19 pandemic period. This may be attributed to the different nature of fear sentiment during the crisis.

\section{Introduction}

The objective of this study is to examine how investor sentiment can predict the volatility of currencies and commodity returns (Smith, 2012). Indeed, during the COVID-19 pandemic, we observe a sense of fear from both investors and individuals alike (Samuel et al., 2020). Since the onset of COVID-19, investor sentiment has been dented, and it is well known that investor sentiment plays an important role in asset pricing (Bouteska, 2019; Cagli et al., 2020; Smales, 2014). Sharma (2020) shows how Asian market volatility has been dynamic and sensitive to the pandemic. This leads us to question whether investor sentiment during the COVID-19 pandemic can predict financial markets. We focus on the predictability of currencies and commodities because during the COVID-19 pandemic, international trade has been strongly impacted due to the state/province/country lockdown policies (Narayan, 2020a; see Phan \& Narayan, 2020; Sha \& Sharma, 2020; Sharma \& Sha, 2020; Vidya \& Prabheesh, 2020). On the other hand, commodities were also heavily impacted, with oil prices decreasing strongly to a negative value on 20 April 2020 at -37.63 USD (see Bajaj, 2020; Narayan et al., 2020). By comparison, gold prices continue to increase, assuming its hedge and safe haven roles (Baur \& Lucey, 2010; Corbet et al., 2019). These developments inspire an investigation of whether investor sentiment can really help predict the volatility of currencies and commodities during the COVID-19 crisis. This is what we seek to understand in this research letter. Subsequently, this is what we contribute to the literature. To-date, when analysing the behaviour of asset prices during the COVID-19 pandemic, studies have shown that: (a) exchange rates (Yen) became more resistance to shocks during the pandemic (Narayan, 2020a); (b) oil prices do not predict the Japanese Yen (Devpura, 2020); bubble activity in exchange rate markets increases significantly during the pandemic (Narayan, 2020b); and the depreciation of Yen had a significant positive effect on returns in the Japanese stock market during the pandemic (Narayan et al., 2020). We contribute to this literature by showing that investor sentiment was in- active in predicting currency and commodity markets.

To achieve the goal of this paper, we use daily data for seven major currency exchange rates (AUD, CAD, CHF, EUR, GBP, JPY, and NZD) against the USD, as well as gold (London) and oil prices (West Texas Intermediate, WTI) over the $01 / 2005-05 / 2020$ period. We then use a GARCH $(1,1)$ model to test if investor sentiment (proxied by the Credit Suisse Fear Barometer (CSFB) and the VIX (more details in Section III)), predicted currency and commodity markets. Our empirical results show that VIX is a better predictor than the $C S F B$ in the whole period and in the global financial crisis (GFC) period. However, they have no predictive power during the COVID-19 pandemic. This result suggests that the COVID-19 pandemic is fundamentally different from the GFC, pointing also to the fact that the nature of investor fear is different during the pandemic compared to the financial crisis.

\section{Data and Methodology}

\section{A. Data}

The CSFB measures the protection against a market crash. It is calculated by selling a $10 \%$ out-of-the-money (OTM) call on the S\&P 500 and using that premium to purchase a downside protection. The level of the index indicates the 0\% OTM strike of the put that makes the strategy net zero cost. A high $C S F B$ signals a high cost of protection relative to upside calls. Therefore, the CSFB measures the option premium which changes in response to the supply and demand for that option. Indeed, buying call options indicates that a trader thinks the market is going higher. Thus, the options being bought and sold indicate that investors are fearful of the potential future risks. So, the higher the $C S F B$, the higher is the fear sentiment of the market.

On the other hand, the VIX is an indicator of the volatility of the US financial market. It is calculated daily by the Chicago Board Option Exchange (CBOE). It is the average of annual volatilities of call and put options on the S\&P 500 . It, thus, measures the fear of investors that is included in the premium of the options on the S\&P 500. The higher the 
$V I X$, the higher is the investor fear.

In addition to the investors' sentiment proxies, our data sample also includes seven major currency exchange rates against the USD, as well as gold prices from the afternoon fixing of the London market, and oil prices (the WTI). Daily data are from $03 / 01 / 2005$ to $08 / 05 / 2020$ and are collected from the Bloomberg terminal.

To make the comparison between the GFC and the COVID-19 crisis periods, we consider three different sample periods. The first one includes the whole period (03/01/ $2005-08 / 05 / 2020)$. The second one is related to the GFC period $(01 / 04 / 2007-05 / 08 / 2011)$, and the third one covers the COVID-19 pandemic period (24/01/2020-08/05/2020). Table 1 presents descriptive statistics of the considered currencies and commodities over the three sub-samples of data.

From Table 1, we can see that only the CAD/USD, JPY/ USD, gold, and oil prices have a positive rate of return over the study period. We also note that the average value is much lower during the COVID-19 pandemic than during the GFC for all the variables. This means that the COVID-19 pandemic has had a stronger effect on the economy than the GFC. As for investor sentiment measures, we note that the value of VIX is much higher than CSFB. Furthermore, their value is much higher during the COVID-19 pandemic than during the GFC. It shows that the COVID-19 pandemic has created a stronger fear sentiment than the GFC.

\section{Methodology}

We follow Smith (2012) and use a two-step estimation of the GARCH $(1,1)$ model in which two lags of investor sentiment (1-day and 5 days) are included in the second step as potential predictors of the volatility of the currency and commodity returns. In the first step, we model daily log returns by the ARCH framework developed by Engle (1982) and Bollerslev (1986). In this model, the conditional variance of the returns is a function of past squared residuals of the return generating process. We consider $a_{t}=r_{t}-\mu_{t}$ as the return innovation on day $t$, with $r_{t}$ the rate of return on day $t$, and $\mu_{t}$ the residual of the return generating process on day $t$. Then, we consider $a_{t}$ which follows a GARCH $(1,1)$ as follows:

$$
a_{t}=\sqrt{h_{t}} \epsilon_{t}, h_{t}=\omega+\alpha a_{t-1}^{2}+\beta h_{t-1}
$$

Where $\epsilon_{t}$ is a sequence of iid random variables with mean 0 and variance $1, \omega>0, \alpha \geq 0, \beta \geq 0$, and $\alpha+\beta<1$. The latter condition implies that the unconditional variance of $a_{t}$ is finite, whereas its conditional variance, $h_{t}$, evolves over time. We estimate the above equation via the method of maximum likelihood with normally distributed errors.

In step 2, we seek to know whether investor sentiment can be a predictor of the volatility of the sampled currencies and commodities. For that, we consider the GARCH $(1,1)$ model defined as follows:

$$
\begin{aligned}
\ln \left(\alpha_{t}^{2}\right)= & \alpha+\beta h_{t}+k_{1} \ln \left(C S F B_{t-1}\right) \\
& +k_{2} \frac{1}{5} \sum_{i=1}^{5} \ln \left(C S F B_{t-i}\right)+z_{t}
\end{aligned}
$$

$$
\begin{aligned}
\ln \left(\alpha_{t}^{2}\right)= & \alpha+\beta h_{t}+k_{3} \ln \left(V I X_{t-1}\right) \\
& +k_{4} \frac{1}{5} \sum_{i=1}^{5} \ln \left(V I X_{t-i}\right)+z_{t}
\end{aligned}
$$

Where $\ln \left(\alpha_{t}^{2}\right)$ are the squared residuals of the mean equation, $r_{t}-\mu_{t} . h_{t}$ is the conditional variance of the returns of the sampled currencies and commodities, as defined in Equation (1). In Equation (2a), $\ln \left(C S F B_{t-1}\right)$ is the short-term predictor representing the $C S F B$ data a day ahead $(t-1)$, and $\frac{1}{5} \sum_{i=1}^{5} \ln \left(C S F B_{t-i}\right)$ is the medium-term predictor representing the 5-days moving average of the CSFB. The same definition is applied in Equation (2b) for $\ln \left(V I X_{t-1}\right)$ and $\frac{1}{5} \sum_{i=1}^{5} \ln \left(V I X_{t-i}\right) ; z_{t}$ is a mean zero disturbance term; $\alpha$ is the intercept term; and $\beta, k_{1}$, and $k_{2}$ (as well as $k_{3}$ and $k_{4}$ ) are parameter estimates for the marginal effects of different factors on the log volatility of the sampled currencies and commodities, $\ln \left(\alpha_{t}^{2}\right)$ :

- $\operatorname{GARCH}(1,1)$ conditional variance with the coefficient $\beta$;

- The predictive power of $C S F B$ is captured by the coefficients $k_{1}$ and $k_{2}$;

- The predictive power the VIX is captured by the coefficients $k_{3}$ and $k_{4}$.

White (1980) standard errors are calculated for the usual tests of significance. Equations (2a) and (2b) are estimated via the maximum likelihood method.

\section{Results}

In Table 2, the results for Equations (2a) and (2b) ${ }^{1}$ for the three considered periods (whole period, GFC period, and the COVID-19 pandemic period) allow us to draw the following observations. First, the $\beta$ coefficient is significant in all cases. This means that the conditional variance of the returns is a good predictor for the volatility of the considered currencies and commodities. Second, the CSFB is not a good predictor of the volatility of the considered currencies and commodities. Indeed, coefficients $k_{1}$ and $k_{2}$ are insignificant in almost all cases (except for EUR and NZD in the whole period, and for $A U D, C A D$, and $C H F$ in the GFC period; and $C A D$ in the COVID-19 pandemic period). Third, the VIX is a good predictor for the volatility of the sampled currencies and commodities, mostly in the medium term, with a lag of five days (except for $C H F, E U R, G B P$, and oil in the GFC period). In the short term, with a lag of one day, the predictive power of the VIX is less efficient, except for CHF and oil in the whole period, and for $A U D, C A D$ and oil in the GFC period. Fourth, in the COVID-19 period, both the CSFB and VIX lose the predictive power in explaining the volatility of the sampled currencies and commodities, except for $C A D$ in the medium term. This result clearly shows that there is a structural change in the currency and commodity markets during the COVID-19 pandemic.

\footnotetext{
1 To save space, the results of Equation (1) based on the ARCH model are not presented here but are available upon request. In the text, we
} would like to focus on the main results related to Equations (2a) and (2b). 
Table 1: Descriptive statistics

\begin{tabular}{|c|c|c|c|c|c|c|c|c|c|c|c|}
\hline \multicolumn{12}{|c|}{ Whole period (03/01/2002-08/05/2020) } \\
\hline & $A U D$ & $C A D$ & $\mathrm{CHF}$ & EUR & GBP & $J P Y$ & $N Z D$ & GOLD & OIL & CSFB & VIX \\
\hline Mean & $-1.75 \mathrm{E}-05$ & $1.39 \mathrm{E}-05$ & $-1.85 \mathrm{E}-05$ & $-2.34 \mathrm{E}-05$ & $-4.46 \mathrm{E}-05$ & $3.75 \mathrm{E}-06$ & $-1.50 \mathrm{E}-05$ & 0.000149 & $7.55 \mathrm{E}-06$ & $4.55 \mathrm{E}-05$ & $5.56 \mathrm{E}-05$ \\
\hline Std. Dev. & 0.00 & 0.00 & 0.00 & 0.00 & 0.00 & 0.00 & 0.00 & 0.00 & 0.01 & 0.01 & 0.03 \\
\hline Skewness & -0.38 & 0.12 & -4.96 & 0.09 & -0.91 & -0.03 & -0.32 & -0.35 & 0.23 & 0.15 & 1.04 \\
\hline \multirow[t]{2}{*}{ Bai \&Ng test } & 1.86 & 3.29 & 1.06 & 4.63 & 1.27 & 3.09 & 2.40 & 3.66 & 2.01 & 2.74 & 2.34 \\
\hline & (0.03) & $(0.00)$ & $(0.14)$ & $(0.00)$ & $(0.10)$ & $(0.00)$ & $(0.00)$ & $(0.00)$ & $(0.02)$ & $(0.00)$ & $(0.00)$ \\
\hline Jarque-Bera & $(0.00)$ & $(0.00)$ & $(0.00)$ & $(0.00)$ & $(0.00)$ & $(0.00)$ & $(0.00)$ & $(0.00)$ & $(0.00)$ & $(0.00)$ & $(0.00)$ \\
\hline \multicolumn{12}{|c|}{ GFC period (01/04/2007-05/08/2011) } \\
\hline & $A U D$ & $C A D$ & $\mathrm{CHF}$ & EUR & $G B P$ & $J P Y$ & $N Z D$ & GOLD & OIL & CSFB & VIX \\
\hline Mean & $8.44 \mathrm{E}-05$ & $-5.77 E-05$ & -0.000182 & $2.26 \mathrm{E}-05$ & $-7.36 \mathrm{E}-05$ & -0.000159 & 4.93E-05 & 0.000361 & 8.02E-05 & -0.000107 & 0.000457 \\
\hline Std. Dev. & 0.00 & 0.00 & 0.00 & 0.00 & 0.00 & 0.00 & 0.00 & 0.00 & 0.01 & 0.01 & 0.03 \\
\hline Skewness & -0.42 & 0.18 & -0.28 & 0.08 & -0.41 & 0.12 & -0.39 & -0.15 & 0.11 & 0.32 & 0.64 \\
\hline Bai \&Ng test & 1.93 & 2.75 & 1.92 & 3.00 & 3.17 & 1.86 & 1.84 & 2.89 & 3.34 & 3.62 & 3.07 \\
\hline Ljung-Box test & $(0.72)$ & $(0.87)$ & $(0.94)$ & $(0.54)$ & $(0.66)$ & $(0.49)$ & $(0.86)$ & $(0.58)$ & $(0.04)$ & (0.01) & $(0.02)$ \\
\hline Jarque-Bera & $(0.00)$ & $(0.00)$ & $(0.00)$ & $(0.00)$ & $(0.00)$ & $(0.00)$ & $(0.00)$ & $(0.00)$ & $(0.00)$ & $(0.00)$ & $(0.00)$ \\
\hline \multicolumn{12}{|c|}{ COVID-19 crisis period (24/01/2020-08/05/2020) } \\
\hline & $A U D$ & $C A D$ & $\mathrm{CHF}$ & EUR & $G B P$ & $J P Y$ & $N Z D$ & GOLD & OIL & CSFB & $V I X$ \\
\hline Mean & -0.000438 & 0.000322 & $-7.36 \mathrm{E}-05$ & -0.000102 & -0.000183 & $-8.92 \mathrm{E}-05$ & -0.000497 & 0.000695 & -0.006318 & -0.001210 & 0.005768 \\
\hline Std. Dev. & 0.00 & 0.00 & 0.00 & 0.00 & 0.00 & 0.00 & 0.00 & 0.00 & 0.02 & 0.03 & 0.05 \\
\hline Skewness & -1.21 & 0.71 & 0.23 & -0.34 & -0.67 & 0.66 & -1.10 & -0.39 & -0.53 & -0.32 & 1.15 \\
\hline \multirow[t]{2}{*}{ Bai \&Ng test } & 1.71 & 1.75 & 1.93 & 2.40 & 3.57 & 1.86 & 1.21 & 2.08 & 3.31 & 3.64 & 3.01 \\
\hline & (0.02) & $(0.00)$ & $(0.02)$ & $(0.00)$ & (0.01) & (0.03) & $(0.03)$ & $(0.00)$ & (0.01) & $(0.00)$ & (0.01) \\
\hline Ljung-Box test & $(0.62)$ & (0.78) & $(0.84)$ & $(0.43)$ & $(0.53)$ & $(0.51)$ & $(0.86)$ & $(0.41)$ & $(0.04)$ & (0.01) & $(0.02)$ \\
\hline Jarque-Bera & $(0.00)$ & $(0.00)$ & (0.01) & $(0.00)$ & (0.00) & $(0.00)$ & $(0.00)$ & (0.00) & $(0.00)$ & $(0.00)$ & $(0.00)$ \\
\hline
\end{tabular}

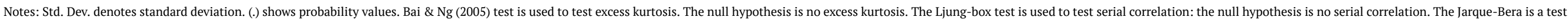

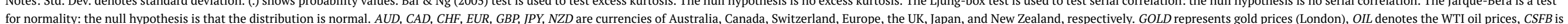
for normality: the null hypothesis is that the distribution is normal. AUD 
Table 2: Results on the predictability of the volatility of currencies and commodities

\begin{tabular}{|c|c|c|c|c|c|c|c|c|c|c|c|c|c|}
\hline \multirow[t]{2}{*}{ Whole period } & \multicolumn{4}{|c|}{ Parameter estimate } & \multicolumn{9}{|c|}{ t-statistics } \\
\hline & $\alpha$ & $\beta$ & $k_{1}$ & $k_{2}$ & $k_{3}$ & $k_{4}$ & $\alpha$ & $\beta$ & $k_{1}$ & $k_{2}$ & $k_{3}$ & $k_{4}$ & Adj. $\mathrm{R}^{2}$ \\
\hline$A U D$ & $-1.20^{* *}$ & $1.01^{* * *}$ & 1.78 & -5.98 & 0.74 & $9.42^{* * *}$ & -1.97 & 19.42 & 0.86 & -1.14 & 0.64 & 3.23 & 0.08 \\
\hline$C A D$ & 0.01 & $1.11^{* * *}$ & 0.65 & 2.44 & 1.57 & $12.13^{* * *}$ & 0.02 & 19.80 & 0.31 & 0.45 & 1.34 & 4.10 & 0.09 \\
\hline $\mathrm{CHF}$ & $-2.74^{* * *}$ & $0.91^{* * *}$ & -0.22 & -6.63 & $-2.47^{* *}$ & $9.41^{* * *}$ & -4.10 & 16.15 & -0.10 & -1.22 & -2.04 & 3.07 & 0.06 \\
\hline EUR & -0.40 & $1.08^{* * *}$ & 0.00 & $-16.75^{* * *}$ & 0.88 & $9.97^{* * *}$ & -0.53 & 17.12 & 0.00 & -2.93 & 0.70 & 3.14 & 0.07 \\
\hline$G B P$ & $-1.68^{* *}$ & $0.98^{* * *}$ & 1.37 & -4.23 & 0.41 & $6.03^{* *}$ & -2.17 & 15.28 & 0.68 & 0.80 & 0.35 & 2.06 & 005 \\
\hline$N Z D$ & -0.32 & $1.09^{* * *}$ & 2.67 & $-10.03^{* *}$ & 0.31 & $11.65^{* * *}$ & -0.45 & 17.53 & -1.31 & -1.93 & 0.27 & 4.03 & 0.07 \\
\hline Gold & $-3.07^{* * *}$ & $0.88^{* * *}$ & 2.82 & 9.08 & -0.38 & $13.58^{* * *}$ & -4.62 & 14.82 & 1.19 & 1.50 & -0.29 & 4.06 & 0.05 \\
\hline Oil & $-1.87^{* * *}$ & $0.97^{* * *}$ & -3.46 & 0.87 & $-3.21^{* *}$ & $12.35^{* * *}$ & -3.84 & 18.77 & -1.50 & 0.14 & -2.49 & 3.97 & 0.08 \\
\hline \multirow[t]{2}{*}{ GFC period } & \multicolumn{4}{|c|}{ Parameter estimate } & \multicolumn{9}{|c|}{$t$-statistics } \\
\hline & $a$ & $\beta$ & $k_{1}$ & $k_{2}$ & $k_{3}$ & $k_{4}$ & $\alpha$ & $\beta$ & $k_{1}$ & $k_{2}$ & $k_{3}$ & $k_{4}$ & Adj. $R^{2}$ \\
\hline$A U D$ & -1.57 & $0.98^{* * *}$ & $9.50^{*}$ & -21.13 & $4.29^{*}$ & $24.76^{* * *}$ & -1.60 & 15.08 & 1.75 & -1.45 & 1.67 & 3.37 & 0.10 \\
\hline$C A D$ & -0.26 & $1.09^{* * *}$ & $14.48^{* * *}$ & 21.90 & $5.99^{* *}$ & $24.81^{* * *}$ & 0.20 & 9.70 & 2.68 & 0.40 & 2.28 & 3.57 & 0.08 \\
\hline EUR & -1.55 & $0.98^{* * *}$ & 3.56 & 1.71 & 4.37 & 9.49 & -1.18 & 8.77 & 0.60 & 1.07 & 1.56 & 1.28 & 0.06 \\
\hline$G B P$ & -1.02 & $0.97^{* * *}$ & 3.43 & -4.49 & 0.32 & 6.09 & 0.40 & 17.39 & 0.63 & 0.18 & 1.02 & 2.11 & 006 \\
\hline$J P Y$ & 0.06 & $1.13^{* * *}$ & 9.57 & 19.32 & 1.86 & $23.34^{* * *}$ & 0.03 & 17.48 & 1.56 & 1.13 & 0.64 & 3.07 & 0.05 \\
\hline$N Z D$ & -0.01 & $1.12^{* * *}$ & 0.50 & 3.88 & 1.29 & $24.90^{* * *}$ & 0.01 & 9.45 & 0.09 & 0.26 & 0.87 & 3.57 & 0.07 \\
\hline Gold & $-4.02^{* * *}$ & $0.77^{* * *}$ & -1.53 & -14.10 & 2.21 & $19.33^{* *}$ & -3.33 & 6.58 & -1.40 & -0.84 & 0.74 & 2.46 & 0.04 \\
\hline Oil & -1.38 & $1.00^{* * *}$ & 7.28 & 17.75 & $5.46^{* *}$ & 10.99 & -1.54 & 10.41 & 1.26 & 1.16 & 2.00 & 0.21 & 0.08 \\
\hline \multirow[t]{2}{*}{ COVID-19 period } & \multicolumn{4}{|c|}{ Parameter estimate } & \multicolumn{9}{|c|}{$t$-statistics } \\
\hline & $a$ & $\beta$ & $k_{1}$ & $k_{2}$ & $k_{3}$ & $k_{4}$ & $a$ & $\beta$ & $k_{1}$ & $k_{2}$ & $k_{3}$ & $k_{4}$ & Adj. $\mathrm{R}^{2}$ \\
\hline$A U D$ & -1.22 & $0.99^{* * *}$ & 9.34 & 31.22 & 3.32 & 0.99 & -1.34 & 13.08 & 0.12 & 0.28 & 0.76 & 0.37 & 0.13 \\
\hline$C A D$ & -0.22 & $1.01^{* * *}$ & 9.30 & $66.60^{* *}$ & 9.95 & 16.57 & -0.10 & 19.30 & 1.38 & 1.96 & 2.21 & 1.17 & 0.22 \\
\hline $\mathrm{CHF}$ & -0.32 & $1.06^{* * *}$ & 12.49 & 30.88 & 4.98 & -10.98 & -0.08 & 3.60 & 1.37 & 0.61 & 0.69 & -0.58 & 0.06 \\
\hline EUR & -4.96 & $0.70^{* *}$ & 6.94 & 11.60 & 4.27 & 9.41 & -1.23 & 1.98 & 1.88 & 0.07 & 1.26 & 1.27 & 0.06 \\
\hline$G B P$ & -1.01 & $0.75^{* * *}$ & 3.13 & -8.49 & 0.12 & 12.53 & 0.20 & 2.15 & 0.37 & 1.18 & 1.37 & 1.09 & 005 \\
\hline$J P Y$ & 0.16 & $0.98^{* * *}$ & 2.27 & 12.31 & 2.84 & 13.34 & 1.03 & 7.42 & 1.16 & 1.03 & 0.23 & 1.27 & 0.13 \\
\hline Oil & -1.18 & $1.09^{*}$ & 7.82 & 7.51 & 5.62 & 0.92 & -1.04 & 0.06 & 1.06 & 0.61 & 0.09 & 0.18 & 0.37 \\
\hline
\end{tabular}

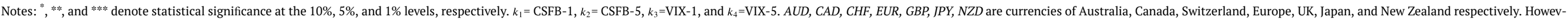
er, GOLD represents gold prices (London), OIL denotes oil prices of WTI, CSFB is Credit Suisse Fear Barometer, and VIX is the financial market volatility. 


\section{Conclusion}

This research letter allows us to conclude that the COVID-19 crisis is of a different nature compared to the GFC, nature. Indeed, during the GFC, investor sentiment, proxied by the Credit Suisse Fear Barometer (CSFB) and the $V I X$, could help predict the volatility of the seven major currency exchange rates against the USD ( $A U D, C A D, C H F$, $E U R, G B P, J P Y, N Z D)$ and of the two major commodities (gold and oil). Furthermore, during the GFC period, the VIX showed a higher predictability power than the CSFB. However, both the CSFB and the VIX lose their predictability power during the COVID-19 crisis. This result suggests that the investor fear sentiment during the two crises is not of the same nature. During the GFC, the fear was mostly relat- ed to the financial loss. However during the COVID-19 crisis, the fear sentiment is strongly related to health issues, in addition to the fear of financial loss due to economic recession. This is why the CSFB and the VIX, proxies of investor fear sentiment related to a financial loss, can help predict the volatility of currencies and commodities during the GFC, but not during the Covid-19 crisis. This finding shows that it is necessary to construct more precise proxies for investor sentiment to be able to distinguish between different natures of fear sentiment.

Submitted: September 25, 2020 AEDT, Accepted: January 03, 2021 AEDT 


\section{REFERENCES}

Bai, J., \& Ng, S. (2005). Tests for skewness, kurtosis, and normality for time series data. Journal of Business \& Economic Statistics, 23(1), 49-60. http s://doi.org/10.1198/073500104000000271

Bajaj, V. (2020, April 22). What negative oil prices mean and how the impact could last. The New York Times.

Baur, D. G., \& Lucey, B. M. (2010). Is gold a hedge or a safe haven? An analysis of stocks, bonds and gold. Financial Review, 45(2), 217-229. https://doi.org/10.1 111/j.1540-6288.2010.00244.x

Bollerslev, T. (1986). Generalized autoregressive conditional heteroskedasticity. Journal of Econometrics, 31(3), 307-327. https://doi.org/10.101 6/0304-4076(86)90063-1

Bouteska, A. (2019). The effect of investor sentiment on market reactions to financial earnings restatements: Lessons from the United States. Journal of Behavioral and Experimental Finance, 24, 100241. https://doi.org/10.1016/j.jbef.2019.100241

Cagli, E. C., Ergün, Z. C., \& Durukan, M. B. (2020). The causal linkages between investor sentiment and excess returns on Borsa Istanbul. Borsa Istanbul Review, 20(3), 214-223. https://doi.org/10.1016/j.bi r.2020.02.001

Corbet, S., Dowling, M., Gao, X., Huang, S., Lucey, B. M., \& Vigne, S. A. (2019). An analysis of the intellectual structure of research on the financial economics of precious metals. Resources Policy, 63, 101416.

Devpura, N. (2020). Can oil prices predict Japanese yen? Asian Economics Letters, 1(3). https://doi.org/1 $\underline{0.46557 / 001 \mathrm{c} .17964}$

Engle, R. F. (1982). Autoregressive conditional heteroscedasticity with estimates of the variance of United Kingdom inflation. Econometrica, 50(4), 987-1007. https://doi.org/10.2307/1912773

Narayan, P. K. (2020a). Has COVID-19 Changed Exchange Rate Resistance to Shocks? Asian Economics Letters, 1(1). https://doi.org/10.46557/001 c.17389

Narayan, P. K. (2020b). Did Bubble Activity Intensify During COVID-19? Asian Economics Letters, 1(2). htt ps://doi.org/10.46557/001c.17654
Narayan, P. K., Devpura, N., \& Wang, H. (2020). Japanese currency and stock market-What happened during the COVID-19 pandemic? Economic Analysis and Policy, 68, 191-198.

Phan, D. H. B., \& Narayan, P. K. (2020). Country responses and the reaction of the stock market to COVID-19-A preliminary exposition. Emerging Markets Finance and Trade, 56(10), 2138-2150. http s://doi.org/10.1080/1540496x.2020.1784719

Samuel, J., Rahman, Md. M., Ali, G. G. M. N., Esawi, E., \& Samuel, Y. (2020). COVID-19 Public Sentiment Insights and Machine Learning for Tweets Classification. Working Paper. https://doi.org/10.3123 4/osf.io/sw2dn

Sha, Y., \& Sharma, S. S. (2020). Research on Pandemics. Emerging Markets Finance and Trade, 56(10), 2133-2137. https://doi.org/10.1080/1540496 x.2020.1795467

Sharma, S. S. (2020). A Note on the Asian Market Volatility During the COVID-19 Pandemic. Asian Economics Letters, 1(2). https://doi.org/10.46557/001 c. 17661

Sharma, S. S., \& Sha, Y. (2020). Part A: Special Section on COVID-19 Research. Emerging Markets Finance and Trade, 56(15), 3551-3553. https://doi.or $\mathrm{g} / 10.1080 / 1540496 x .2020 .1858617$

Smales, L. A. (2014). News sentiment and the investor fear gauge. Finance Research Letters, 11(2), 122-130. https://doi.org/10.1016/i.frl.2013.07.003

Smith, G. P. (2012). Google Internet search activity and volatility prediction in the market for foreign currency. Finance Research Letters, 9(2), 103-110. htt ps://doi.org/10.1016/i.frl.2012.03.003

Vidya, C. T., \& Prabheesh, K. P. (2020). Implications of COVID-19 pandemic on the global trade networks. Emerging Markets Finance and Trade, 56(10), 2408-2421. https://doi.org/10.1080/1540496x.2020.1 $\underline{785426}$

White, H. (1980). A Heteroskedasticity-Consistent Covariance Matrix Estimator and a Direct Test for Heteroskedasticity. Econometrica, 48(4), 817-838. htt ps://doi.org/10.2307/1912934 